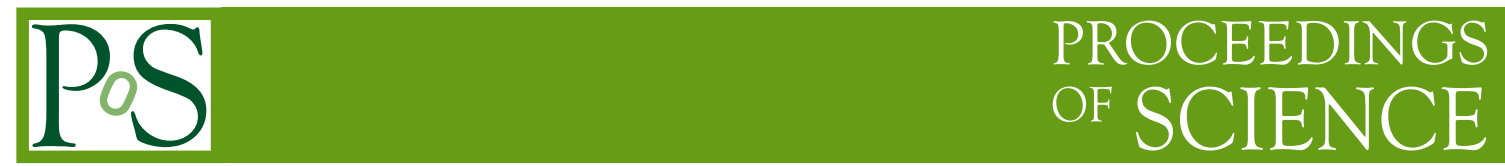

\title{
Overlap fermion in external gravity
}

\author{
Hiroto So ${ }^{* \dagger}$ \\ Department of Physics, Niigata University, Ikarashi 2-8050, Niigata, 950-2181, Japan \\ E-mail: so@muse.sc.niigata-u.ac.jp
}

\section{Masashi Hayakawa}

Department of Physics, Nagoya University, Nagoya 464-8602, Japan

E-mail: hayakawa@eken.phys.nagoya-u.ac.jp

\section{Hiroshi Suzuki}

Theoretical Physics Laboratory, RIKEN, Wako 2-1, Saitama 351-0198, Japan

E-mail: hsuzuki@riken.jp

On a lattice, we construct an overlap Dirac operator which describes the propagation of a Dirac fermion in external gravity. The local Lorentz symmetry is manifestly realized as a lattice gauge symmetry, while the general coordinate invariance is expected to be restored only in the continuum limit. The lattice index density in the presence of a gravitational field is calculated.

XXIV International Symposium on Lattice Field Theory

July 23-28 2006

Tucson Arizona, US

\footnotetext{
* Speaker.

${ }^{\dagger}$ After October 1st in 2006, the e-mail address is changed to so@phys.sci.ehime-u.ac.jp .
} 


\section{Motivations}

Lattice gauge theory is not just a regularized theory but a real calculable method for infinite degrees of freedom. There are two successes in the theory; exact gauge symmetry on lattice and nonperturbative calculation of gauge dynamics. Nevertheless, lattice fermion problems such as doubling and the chiral symmetry realization were unsolved. Afterwards, a new approach by an overlap fermion or Ginsparg-Wilson fermion brings to us two successes [1, 2, 3]; involving an exact chiral symmetry [4] and anomaly calculation [5, 6, 8, 9]. One may have a question: Can we involve gravity interaction and calculate the chiral anomaly on lattice?

Usual approaches of the lattice gravity are Regge calculus and random lattice method[10]. We mainly treat only an external gravity and use a fixed lattice(the spacing and the structure). So, our setting is the following; the lattice is spacing-fixed and cubic-connected, The coordinate is defined as just an integer $x$, which is no real scale, gravity interaction is involved as a spin connection, $U(x, \mu) \in \operatorname{spin}(4)$. Another gravity field is denoted by a vierbein $e_{\mu}^{a}(x) \in \mathrm{GL}(4, R)$. Different from ordinary gauge symmetry cases, we must consider a nontrivial hermiticity (a metric) condition. As the result, the spin connection is connected with the vierbein field. A serious problem of our method is the continuum limit. Although the local Lorentz invariance is kept as exact lattice invariance, the general coordinate invariance is expected after the continuum limit. In the formulation, can we calculate the chiral anomaly? This is not a trivial problem. In this talk, the formulation of lattice overlap fermion with an external gravity and the calculation of the chiral anomaly are sketched. For a detail description, see ref. [11]. In addition, an idea for a dynamical gravity is presented.

\section{Lattice formulation with local Lorentz invariance}

We start with a lattice Dirac operator which is defined in terms of the nearest-neighbor forward covariant difference:

$$
\not \nabla \psi(x) \equiv \sum_{\mu} \gamma^{\mu}(x)\{U(x, \mu) \psi(x+\hat{\mu})-\psi(x)\}
$$

This behaves covariantly, i.e., $\not \nabla \psi(x) \rightarrow g(x) \not \nabla \psi(x)$, under the local Lorentz transformation

$$
\begin{aligned}
& \psi(x) \rightarrow g(x) \psi(x), \quad \bar{\psi}(x) \rightarrow \bar{\psi}(x) g(x)^{-1}, \\
& U(x, \mu) \rightarrow g(x) U(x, \mu) g(x+\hat{\mu})^{-1}, \quad \gamma^{\mu}(x) \rightarrow g(x) \gamma^{\mu}(x) g(x)^{-1},
\end{aligned}
$$

where $\gamma^{\mu}(x) \equiv \sum_{a} e_{a}^{\mu}(x) \gamma^{a}$ and $g(x) \in \operatorname{spin}(4)$. The naive continuum limit of $\not \nabla$ coincides with the Dirac operator in the continuum, denoted by $\not \supset$. To ensure that the lattice index theorem holds in lattice gauge theory, in addition to the Ginsparg-Wilson relation, the $\gamma_{5}$ hermiticity of a lattice Dirac operator is very important. We are therefore naturally led to attempt to clarify the meaning of hermiticity in the presence of a gravitational field.

We introduce the inner product of two functions on the lattice with spinor indices as

$$
(f, g) \equiv \sum_{x} e(x) f(x)^{T *} g(x)
$$


by using the determinant of the vierbein, $e(x)$. The inner product (2.3) is a natural lattice counterpart of the general coordinate invariant inner product in a curved space. Next, we note

$$
(f, \not \nabla g)=-\left(\not \nabla^{*} f, g\right)-\sum_{x}\left\{\sum_{\mu} \nabla_{\mu}^{*}\left\{e(x) \gamma^{\mu}(x)\right\} f(x)\right\}^{T *} g(x),
$$

where a lattice Dirac operator is defined in terms of the backward covariant difference,

$$
\begin{aligned}
& \not{ }^{*} \psi(x) \equiv \sum_{\mu} e(x)^{-1} e(x-\hat{\mu}) U(x-\hat{\mu}, \mu)^{-1} \gamma^{\mu}(x-\hat{\mu}) U(x-\hat{\mu}, \mu) \\
& \times\left\{\psi(x)-U(x-\hat{\mu}, \mu)^{-1} \psi(x-\hat{\mu})\right\},
\end{aligned}
$$

and the covariant divergence of $e(x) \gamma^{\mu}(x)$ is written as

$$
\sum_{\mu} \nabla_{\mu}^{*}\left\{e(x) \gamma^{\mu}(x)\right\} \equiv \sum_{\mu}\left\{e(x) \gamma^{\mu}(x)-e(x-\hat{\mu}) U(x-\hat{\mu}, \mu)^{-1} \gamma^{\mu}(x-\hat{\mu}) U(x-\hat{\mu}, \mu)\right\} .
$$

(Note that $\gamma^{a}$ and $\gamma^{\mu}(x)$ do not commute with the link variables $U(x, \mu)$, i.e., $\left[\gamma^{\mu}(x), U(y, v)\right] \neq 0$.) The $\not \nabla^{*}$ also behaves covariantly under the local Lorentz transformation and coincides with the continuum Dirac operator $\not \supset$ in the naive continuum limit. The equality 2.4 shows that, if we postulate

$$
\sum_{\mu} \nabla_{\mu}^{*}\left\{e(x) \gamma^{\mu}(x)\right\}=0, \quad \text { for all } x
$$

on the external vierbein and the link variables as the lattice counterpart of the metric condition, then the $\not \nabla^{*}$ in eq. (2.5) is precisely the minus hermitian conjugate of $\not \nabla$ in eq. (2.1):

$$
\not \not^{*}=-\not^{\dagger}, \quad\left(\not \nabla^{*}\right)^{\dagger}=-\not \nabla .
$$

Having clarified the meaning of the hermitian conjugation, it is straightforward to construct a lattice Dirac operator of overlap type with the desired properties. We first define the Wilson-Dirac operator by

$$
D_{\mathrm{w}}=\frac{1}{2}\left\{\not \nabla+\not \nabla^{*}-\frac{1}{2}\left(\not \not^{*} \not \nabla+\not \nabla \not{ }^{*}\right)\right\}
$$

where the second term is the Wilson term in a curved space, which is hermitian with respect to the inner product (2.3). Then in analogy to the case of lattice gauge theory, we define the overlap-Dirac operator from the Wilson-Dirac operator as

$$
D=1-A\left(A^{\dagger} A\right)^{-1 / 2}, \quad A \equiv 1-D_{\mathrm{w}} .
$$

The naive continuum limit of the operator $D$ is $\not \supset$. It is easy to confirm that the free Dirac operator does not suffer from species doubling.

From its construction (2.10) and the $\gamma_{5}$ hermiticity of the Wilson-Dirac operator, we find that the operator $D$ satisfies the Ginsparg-Wilson relation in the conventional form,

$$
\gamma_{5} D+D \gamma_{5}=D \gamma_{5} D
$$

and that it is also $\gamma_{5}$ hermitian, i.e.,

$$
D^{\dagger}=\gamma_{5} D \gamma_{5},
$$

with respect to the inner product (2.3). 


\section{Calculation of lattice index density}

We have defined a lattice Dirac operator of overlap type that describes the propagation of a single Dirac fermion in external gravitational fields. Since the forms of the Ginsparg-Wilson relation and the $\gamma_{5}$ hermiticity are identical to those in lattice gauge theory, we can repeat the same argument for the index theorem in the latter theory.

A natural lattice action for the massless Dirac fermion in a curved space is

$$
S_{\mathrm{F}}=\sum_{x} e(x) \bar{\psi}(x) D \psi(x) .
$$

This action is invariant under the modified chiral transformation

$$
\psi(x) \rightarrow\left(1+i \theta \hat{\gamma}_{5}\right) \psi(x), \quad \bar{\psi}(x) \rightarrow \bar{\psi}(x)\left(1+i \theta \gamma_{5}\right),
$$

where $\theta$ is an infinitesimal constant parameter and $\hat{\gamma}_{5} \equiv \gamma_{5}(1-D)$, due to the Ginsparg-Wilson relation (2.11). The functional integration measure is, however, not invariant under this transformation and gives rise to a non-trivial Jacobian $J$, where

$$
\ln J=-2 i \theta \sum_{x} \operatorname{tr} \Gamma_{5}(x, x)
$$

and

$$
\Gamma_{5}(x, y) \equiv \gamma_{5}\left(\delta_{x y}-\frac{1}{2} D(x, y)\right) .
$$

The operator $\Gamma_{5}$ anti-commutes with the hermitian operator $H \equiv \gamma_{5} D$, again due to the GinspargWilson relation. It is then easy to prove the lattice index theorem. The quantity

$$
\sum_{x} \operatorname{tr} \Gamma_{5}(x, x)=n_{+}-n_{-},
$$

where $n_{ \pm}$denote the numbers of zero eigenmodes of the $H$ with positive and negative chiralities, respectively, is an integer even for finite lattice spacings. This is the lattice index theorem in the presence of a gravitational field.

We next consider the classical continuum limit of the density of the lattice index (3.5), which is precisely the chiral anomaly. Although the direct calculation is quite involved, we can resort to the following argument which utilizes the topological nature of the lattice index. First we note that in the expressions of the index, $\Gamma_{5}$ can be replaced by $\Gamma_{5} e^{-H^{2} / M^{2}}$ with an arbitrary mass $M$, because only zero eigenmodes of $H$ contribute to the index. Namely, we have

$$
\sum_{x} \operatorname{tr} \Gamma_{5}(x, x)=\sum_{x} \operatorname{tr} \sum_{y} \Gamma_{5} e^{-H^{2} / M^{2}}(x, y) \delta_{y x} \equiv a^{4} \sum_{x} \mathscr{A}_{5}(x),
$$

where the lattice spacing $a$ has been restored, and the index density has been defined by

$$
\mathscr{A}_{5}(x) \equiv \frac{1}{a^{4}} \operatorname{tr} \sum_{y} \Gamma_{5} e^{-H^{2} / M^{2}}(x, y) \delta_{y x} .
$$

From this point, we consider the index density on a lattice with infinite extent, as usual for the classical continuum limit. Then we have

$$
\mathscr{A}_{5}(x)=\int_{\mathscr{B}} \frac{\mathrm{d}^{4} k}{(2 \pi)^{4}} e^{-i k x} \operatorname{tr}\left(\gamma_{5}-\frac{a}{2} H\right) e^{-H^{2} / M^{2}} e^{i k x},
$$


where $\mathscr{B}$ denotes the Brillouin zone. Then using the fact that the lattice free Dirac operator does not possess doubler's zero, and $e^{-H^{2} / M^{2}}$ acts as a suppression factor at the boundary of the Brillouin zone for $a \rightarrow 0$, we can show that

$$
\begin{aligned}
\lim _{a \rightarrow 0} \mathscr{A}_{5}(x) & =\int_{\mathbb{R}^{4}} \frac{\mathrm{d}^{4} k}{(2 \pi)^{4}} \lim _{a \rightarrow 0} e^{-i k x} \operatorname{tr}\left(\gamma_{5}-\frac{a}{2} H\right) e^{-H^{2} / M^{2}} e^{i k x} \\
& =\int_{\mathbb{R}^{4}} \frac{\mathrm{d}^{4} k}{(2 \pi)^{4}} e^{-i k x} \operatorname{tr} \gamma_{5} e^{\not D^{2} / M^{2}} e^{i k x},
\end{aligned}
$$

because $\lim _{a \rightarrow 0} H=\gamma_{5} \not D$ in the naive continuum limit. It is interesting that, in this calculational scheme [12], the term proportional to $a H / 2$ does not contribute. In this way, we obtain the index density in the continuum theory. Namely,

$$
\lim _{a \rightarrow 0} a^{4} \sum_{x} \operatorname{tr} \Gamma_{5}(x, x)=\int_{M_{4}} \lim _{a \rightarrow 0} \mathscr{A}_{5}(x)=\int_{M_{4}} \operatorname{det}\left\{\frac{i \hat{R} / 4 \pi}{\sinh (i \hat{R} / 4 \pi)}\right\}^{1 / 2},
$$

where the curvature 2-form is defined by $(\hat{R})_{a}^{b}=\frac{1}{2} R_{\mu v a}{ }^{b} \mathrm{~d} x^{\mu} \wedge \mathrm{d} x^{\nu}$. Thus as expected from the absence of the species doubling (which implies the correct number of degrees of freedom) and the topological properties of the lattice index, we find that our formulation reproduces the correct expression of the chiral $\mathrm{U}(1)$ anomaly in a gravitational field. This demonstration can be regarded as a test of the restoration of the general coordinate invariance in the classical continuum limit.

We can also generalize our construction to other Lorentz (reducible) representations. By a similar argument to the above, we have, for the spinor-vector representation,

$$
\lim _{a \rightarrow 0} \sum_{x} \operatorname{tr} \Gamma_{5}(x, x)=\int_{M_{4}} \operatorname{det}\left\{\frac{i \hat{R} / 4 \pi}{\sinh (i \hat{R} / 4 \pi)}\right\}^{1 / 2} \operatorname{tr}\left\{e^{-i \hat{R} /(2 \pi)}\right\}
$$

and, for the bi-spinor representation

$$
\lim _{a \rightarrow 0} \sum_{x} \operatorname{tr} \Gamma_{5}(x, x)=\int_{M_{4}} \operatorname{det}\left\{\frac{2 i \hat{R} / 4 \pi}{\tanh (i \hat{R} / 4 \pi)}\right\}^{1 / 2} .
$$

These are well-known expressions of the index for those representations.

\section{Summary}

We formulated an overlap fermion on lattice with an external gravity and calculated a chiral index density. In the formulation, several essential points are listed as (i) the local Lorentz symmetry is realized as the exact lattice gauge symmetry; (ii) the general coordinate invariance is restored after the continuum limit; (iii) a hermiticity condition of conjugate difference operators is important; (iv) a Wilson-Dirac operator is constructed in advance, and (v) an overlap-Dirac operator is constructed and the Ginsparg-Wilson relation is verified; (vi) the calculation of the chiral index density can be executed not only for Dirac fermions but also for other representations.

Validity of this formulation depend on the continuum limit strongly. If this continuum limit or the fixed point has the property of a general covariance, a next step is to involve the dynamics of gravity. The limit is expected to hold a relation,

$$
\left\langle e_{\mu}^{a}(x) e_{v}^{a}(x)\right\rangle=g_{\mu v}(x),
$$


where $\langle\cdots\rangle$ means an average under the dynamics of vierbein fields to fix a physical scale(distance). Vierbein fields generally express a connection between a curved space and the tangent space. Although we treat only a lattice tangent space, a real curved space may be realized after the dynamics of vierbein fields and some smoothing of the tangent space.

\section{Acknowledgments}

This work is supported in part by the Grants-in-Aid for Scientific Research Nos. 13135203, 13135223, 17540242, 18034011 and 18540305 from the Japan Society for the Promotion of Science.

\section{References}

[1] P. H. Ginsparg and K. G. Wilson, A remnant of chiral symmetry on the lattice, Phys. Rev. D 25 (1982) 2649.

[2] H. Neuberger, Exactly massless quarks on the lattice, Phys. Lett. B 417 (1998) 141 [hep-lat/9707022]; More about exactly massless quarks on the lattice, Phys. Lett. B 427 (1998) 353 [hep-lat/9801031].

[3] R. Narayanan, Ginsparg-Wilson relation and the overlap formula, Phys. Rev. D 58 (1998) 097501 [hep-lat/9802018].

[4] M. Lüscher, Exact chiral symmetry on the lattice and the Ginsparg-Wilson relation, Phys. Lett. B 428 (1998) 342 [hep-lat/9802011].

[5] P. Hasenfratz, Lattice QCD without tuning, mixing and current renormalization, Nucl. Phys. B 525 (1998) 401 [hep-lat/9802007].

P. Hasenfratz, V. Laliena and F. Niedermayer, The index theorem in QCD with a finite cut-off, Phys. Lett. B 427 (1998) 125 [hep-lat/9801021].

[6] Y. Kikukawa and A. Yamada, Weak coupling expansion of massless QCD with a Ginsparg-Wilson fermion and axial U(1) anomaly, Phys. Lett. B 448 (1999) 265 [hep-lat/9806013].

[7] D. H. Adams, Axial anomaly and topological charge in lattice gauge theory with overlap-Dirac operator, Ann. of Phys. 296 (2002) 131 [hep-lat/9812003].

[8] H. Suzuki, Simple evaluation of chiral Jacobian with the overlap Dirac operator, Prog. Theor. Phys. 102 (1999) 141 [hep-th/9812019].

[9] T. Fujiwara, K. Nagao and H. Suzuki, Axial anomaly with the overlap-Dirac operator in arbitrary dimensions, JHEP 09 (2002) 025 [hep-lat/0208057].

[10] R. Sorkin, Time evolution problem in Regge calculus, Phys. Rev. D 12 (1975) 385; The electromagnetic field on a simplicial net, J. Math. Phys. 16 (1975) 2432 [Errata; 19 (1978) 1800]. T. Banks, Y. Dothan and D. Horn, Geometric fermions, Phys. Lett. B 117 (1982) 413. N. P. Warner, The application of Regge calculus to quantum gravity and quantum field theory in a curved background, Proc. R. Soc. London A 383 (1982) 359.

M. Shimono, Fermions and gravitational anomaly in lattice gravity, Prog. Theor. Phys. 84 (1990) 331. N. Kawamoto and H. B. Nielsen, Lattice gauge gravity with fermions, Phys. Rev. D 43 (1991) 1150. B. A. Berg and B. Krishnan, Phase structure of $\mathrm{SU}(2)$ lattice gauge theory with quantum gravity, Phys. Lett. B 318 (1993) 59 [hep-lat/9306006]. 
J. Ambjørn, K. N. Anagnostopoulos and J. Jurkiewicz, Abelian gauge fields coupled to simplicial quantum gravity, JHEP 08 (1999) 016 [hep-lat/9907027].

$\mathrm{S}$. N. Vergeles, The lattice quantum gravity, its continuum limit and the cosmological constant problem, hep-th/0411096.

[11] M. Hayakawa, H. So and H. Suzuki, Overlap lattice fermion in a gravitational field, Prog. Theor. Phys. 116 (2006) 197 [hep-lat/0604003].

[12] K. Fujikawa, A continuum limit of the chiral Jacobian in lattice gauge theory, Nucl. Phys. B 546 (1999) 480 [hep-th/9811235]. 